\title{
Stereoelectroencephalography versus Subdural Electrode Implantation to Determine Whether Patients with Drug-resistant Epilepsy Are Candidates for Epilepsy Surgery
}

\author{
Brian FIANI, ${ }^{1}$ Ryan JARRAH, ${ }^{2}$ Thao DOAN, ${ }^{3}$ Jennifer SHIELDS, ${ }^{4}$ \\ Rebecca HOUSTON, ${ }^{1}$ and Erika SARNO ${ }^{5}$ \\ ${ }^{1}$ Department of Neurosurgery, Desert Regional Medical Center, Palm Springs, CA, USA \\ ${ }^{2}$ University of Michigan - Flint, Flint, MI, USA \\ ${ }^{3}$ University of Texas Medical Branch, Galveston, TX, USA \\ ${ }^{4}$ Michigan State University College of Human Medicine, East Lansing, MI, USA \\ ${ }^{5}$ Michigan State University College of Osteopathic Medicine, East Lansing, MI, USA
}

\begin{abstract}
Epilepsy is a chronic condition that affects about 50 million individuals worldwide. While its challenges are profound, there are increasing instances where antiepileptic drugs (AEDs) fail to provide relief to epileptic manifestations. For these pharmacoresistant cases, epilepsy surgery often is an effective route for treatment. However, the complexity and challenges associated with presurgical evaluations have prevented more widespread utilization of epilepsy surgery in pharmacoresistant cases. While preliminary work-ups and non-invasive diagnostic imaging have allowed for limited identification of the epileptogenic zone (EZ), there is yet to be an established pre-determined algorithm for surgical evaluation of patients with epilepsy. However, two modalities are currently being used for localization of the EZ and in determining candidates for surgery: stereoelectroencephalography (SEEG) and subdural electrodes (SDEs). SDE has been used in the United States for decades; however, SEEG now provides a less invasive option for mapping brain regions. We seek to address which intracranial monitoring technique is superior. Through a review of the outcomes of various clinical studies, SEEG was found to have greater safety and efficiency benefits than SDE, such as lower morbidity rates, lower prevalence of neurological deficits, and shorter recovery times. Moreover, SEEG was also found to have further functional benefits by allowing for deeper targeting of cerebral tissue along with bilateral hemispheric monitoring. This has led to increased rates of seizure freedom and control among SEEG patients. Nevertheless, further studies on the limitations and advancements of SEEG and SDE are still required to provide a more comprehensive understanding regarding their application.
\end{abstract}

Keywords: intracranial EEG, stereoelectroencephalography, epilepsy surgery, drug-resistant epilepsy, subdural monitoring

\section{Introduction}

Epilepsy is a chronic condition characterized by the predisposition of the brain to generate recurrent

Received October 25, 2020; Accepted February 16, 2021

Copyright $\subseteq 2021$ by The Japan Neurosurgical Society This work is licensed under a Creative Commons AttributionNonCommercial-NoDerivatives International License. seizures, unprovoked by any immediate stimulus. ${ }^{1)}$ According to the Center for Disease Control (CDC), active epilepsy in adults ( $>18$ years of age) is characterized by a history of doctor-diagnosed epilepsy in a patient who is (a) currently taking medication or (b) has had one or more seizures in the past year. ${ }^{2)}$ Based on this definition, in 2015, 3 million adults in the United States had active epilepsy. ${ }^{2}$ Worldwide, the total number of individuals affected is estimated to be 50 million. ${ }^{3)}$ Anti-seizure drugs 
(ASDs) are routinely used as a first-line pharmacotherapies for seizure control. However, one-third of patients still suffer from uncontrolled seizures despite pharmacotherapy. ${ }^{4,5)}$ Fortunately, epilepsy surgery can significantly improve patients with drug-resistant epilepsy (DRE) and possibly eradicate manifesting seizure.5) A randomized control study (RCT) comparing surgical and pharmacological ASD treatment outcomes in temporal lobe epilepsy (TLE) showed, after 1-year follow-up, 58\% of patients in the surgery group were seizure-free compared to $8 \%$ in the ASD group. ${ }^{6}$ Other studies have demonstrated the effectiveness of various surgical methods, although risk of cognitive deficits and decreased effectiveness with increased time post-operation remain key concerns..$^{7-9)}$

Unfortunately, epilepsy surgery remains underutilized due to some degree to the complexity of presurgical evaluation. ${ }^{5}$ However, lack of physician recognition of this technique and limited patient access to epilepsy centers play a major role in the underutilization of epilepsy surgery. Before devising a surgical plan, a series of tests must identify the seizure type, localize the seizure onset focus, or epileptogenic zone (EZ), and confirm the safety of the surgery to deem a patient as a suitable candidate. Accurate and safe localization of the EZ is of particular importance. Initially, non-invasive neuroimaging, electroencephalographic, and clinical data are used to delineate the region. If these modalities are inconclusive, direct intracranial recordings are needed. Stereoelectroencephalography (SEEG) and subdural electrodes (SDEs) are the two most common methods for recording directly from the cortex to identify the EZ. ${ }^{10)}$ In the United States, SDE has been the predominant method, while SEEG has been more popular in France, Italy, and some Canadian epilepsy centers. ${ }^{10,11)}$ One possible explanation for the lag time in the United States is that SEEG literature was not widely disseminated in English creating a language barrier. Moreover, the delayed integration could be due to conceptual differences. This includes a difference in surgical philosophy and approach, as SDE has long been utilized as the standard of care for epileptic monitoring. The introduction of an advanced model such as SEEG would bring a learning curve that may be deemed unconventional to the surgeon. In addition, the technical aspects of SEEG are associated with increased demands on epilepsy surgery teams compared to other techniques delaying its implementation. ${ }^{1)}$ The complexity of invasive monitoring and heterogeneity of the epilepsy patient population makes it difficult to conduct clinical trials comparing the two techniques. However, there are plenty of observational studies that demonstrate the safety and drawbacks of each technique. The objective of this manuscript is to provide a review of the current literature on outcome differences, including safety and efficacy, of SEEG and SDE. This will help inform clinical decision-making when choosing an invasive monitoring strategy for delineation of the EZ prior to epilepsy surgery.

\section{Clinical Presentation}

Epilepsy is characterized by recurrent, unprovoked seizures. Clinically, it may present as alterations in awareness, behavior, and cognitive capacity, in addition to dysfunction of the motor, sensory, or autonomic systems. ${ }^{12)}$ Clinical manifestations of epilepsy may vary contingent on the underlying cause, the patient's age, the cortical regions perturbed by abnormal neuronal activity, and the configuration in which the neuronal discharge permeates. ${ }^{12)}$ Such features determine the type of seizure, which can be categorized as either generalized or focal and then further classified. Generalized seizures affect regions in both cerebral hemispheres; there are six types, including absence (petit mal), clonic, tonic, tonic-clonic (grand-mal), atonic, and myotonic seizures. ${ }^{12)}$ In contrast, focal (partial) seizures impact a localized area of the brain; these can be divided into focal awareness or impaired awareness. ${ }^{12)}$ Moreover, focal seizures can evolve into generalized seizures. Impending seizure activity may be signaled by preceding unusual sensations, referred to as an aura, which may include sensory, physical, or emotional changes, such as visual disturbances, abnormal smells, numbness or tingling, nausea, or a sense of fear or anxiety.

\section{Natural History}

Little is known of the natural course of untreated epilepsy, as most studies investigating epilepsy outcomes are centered around treatment. ${ }^{13-15)}$ Findings from few, small-scale studies, most performed in resource-poor countries with limited healthcare access, provide circumstantial evidence that a portion of cases of untreated epilepsy enter spontaneous remission, varying from up to $20 \%$ to $50 \%$ of cases. ${ }^{13-15)}$

In developed countries, treatment is more easily accessible and, therefore, often started immediately following diagnosis. Customarily, pharmacotherapy is the first-line management for epilepsy. Antiepileptic drugs (AEDs) are effective in achieving sustained seizure-freedom for about two-thirds of the patient population, meaning that these patients do not have a seizure for whichever is period longer: thrice the 
time of their longest inter-seizure interval or 1 year. ${ }^{5)}$ Of these individuals, $47 \%$ are completely seizurefree with the use of their first AED. ${ }^{5)}$ The probability of seizure-freedom decreases with each unsuccessful AED; only $13 \%$ may achieve seizure remission with a second AED, and a mere $4 \%$ using a third agent or combination therapy. ${ }^{5}$ Thus, more than one-third of patients develop DRE, meaning they have tried and failed at least two adequate AED trials of aptly chosen and tolerated mono or polytherapies administered at therapeutic doses. ${ }^{5,16,17)}$ Drug resistance in epilepsy significantly increases injury rates, risk of disability, morbidity and mortality, as well as reduces quality of life and life expectancy. ${ }^{17,18)}$ These patients may qualify as candidates for epilepsy surgery, an underutilized but effective standard of care, and possibly the only means to cure DRE for most. ${ }^{5,16-20)}$ Consideration for surgical intervention requires a referral to a comprehensive epilepsy program consisting of a multidisciplinary team of specialists who perform an extensive presurgical evaluation; and this ultimately determines surgical eligibility, the type of surgical procedures available, and other possible supplementary treatment methods. ${ }^{5,17,19)}$ Resistance to AEDs is the initial indication for surgical intervention, in addition to the requirement that the seizures are disabling. ${ }^{19)}$ Further indications may include patients with complex epilepsy syndromes or those mandating complex surgical procedures; patients with seizures showing stereotyped, lateralizing, or focal findings but lack a defined electroclinical syndrome; and pediatric patients with a magnetic resonance imaging (MRI)-confirmed lesion responsive to surgical resection. ${ }^{19)}$

\section{Imaging and work-up for surgical referral}

There does not exist a pre-determined algorithm for timing and type of imaging and presurgical work-up for epilepsy surgery. The absence of an agreed upon surgical evaluation is a reflection of the highly diverse collection of diseases that manifest in epileptic seizures coupled with the complexity of preoperative work-up in the context of the challenging nature of the American healthcare system. Provider preferences, institutional availability, socioeconomics, and factors unique to each clinical case also play a role. ${ }^{21,22)}$ Regardless, once a patient meets the defined criteria for DRE as determined by the American Academy of Neurology (AAN) and International League Against Epilepsy (ILAE), they should be referred to an epilepsy center for further work-up and surgical evaluation. ${ }^{21,23)}$ This is a critical point, as studies have shown an unacceptable and dangerously long 20-year lag between epilepsy diagnosis and surgical referral for adults in the United States. ${ }^{24)}$
Preliminary work-up includes a detailed history and physical examination, 3-Tesla (3T) or higher MRI brain with and without contrast, evaluation of clinical semiology, interictal EEG, and video EEG (vEEG) to evaluate for localization of a seizure focus. Beyond a record of semiology, history should include onset of epilepsy/duration, seizure risk factors, frequency, and associated symptoms. Notably, lack of any seizure focus, or on the other hand, identification of multiple possible seizure foci have been cited as a common reason for the termination of surgical workup. However, neither negative MRI, multifocal MRI, nor lack of localizing data from vEEG should result in disqualification from further surgical evaluation. ${ }^{21)}$ There are multiple non-medical treatment options for treatment of multifocal, generalized epilepsy, and for those with foci in eloquent regions. ${ }^{22}$ An appropriate preoperative evaluation that is both comprehensive and germane is essential to achieving the best possible outcomes for all DRE patients.

Interictal positron emission tomography (PET) scan may confirm EZ and may be used in evaluation of patients with no EZ lesion seen on MRI. LoPinto-Khoury et al. compared surgical outcomes for patients whose MRIs were non-lesional (including negative for mesial temporal sclerosis [MTS]) but whose PET scans demonstrated areas of hypometabolism in the anteromedial temporal lobe (PET+/MRI-) to those with demonstrated MTS on MRI. They found that $75 \%$ of PET+/MRI- patients who underwent epilepsy surgery achieved Class I Engel Surgical Outcomes and thus were free of disabling seizures postoperatively. ${ }^{25)}$ 5-year outcomes for this group were comparable to outcomes of MTS (MRI+) patients. Neuropsychological testing may also contribute to identification of an EZ, establish a baseline for future evaluation, and assess aspects of neurocognitive performance that may be impacted by surgical intervention.

Further testing is variable depending on the specifics of the case. Ictal single-photon emission computed tomography (SPECT) may identify areas of hyperperfusion while subtraction of ictalinterictal SPECT co-registered to MRI (SISCOM) has been shown to provide high sensitivity and specificity for identification of an EZ, particularly in temporal lobe locations in both adult and pediatric patients. ${ }^{26,27)}$ This technique is limited by the resource-intensiveness implicit to obtaining ictal studies. MEG or functional MRI (fMRI) may have utility in surgical planning by way of providing identification of eloquent cortex. Wada testing may be used for lateralization of memory and language functions. 
Invasive monitoring may be performed on patients for whom a possible EZ area is identified by not confirmed during non-invasive testing. For patients with non-lateralized or likely deep EZ(s), SEEG may be performed, whereas patients with an inexplicit EZ on SEEG or who require mapping of eloquent cortex may benefit from SDE. Indications, features, and outcomes associated with SEEG and SDE are discussed in further detail below.

\section{SEEG}

\section{Novel nuances}

SEEG is a method used to identify EZ zone for possible surgical intervention in patients with DRE. The EZ is a theoretical concept that defines the cortical area that generates seizures, and it is the target in epilepsy surgery to stop seizures. ${ }^{28)}$ SEEG was originally developed by Jean Talairach and Jean Bancaud in France during the 1950s. ${ }^{29)}$ The original SEEG method by Talairach was complex and used a stereotactic frame with a double grid system. ${ }^{28,30)}$ While the first stereotactic frames invented were limited to analyzing the cortical surface, Talairach and Bancaud wanted to implement an indirect method to analyze the cerebral cortex and deep gray nuclei of the brain in relation to visible structures. Talairach recognized the anatomical variability among different patients, so they developed a proportional grid system. This new system meant the entire volume of the brain could be analyzed in comparison with stereotactic frames that solely focused on specific lobes or deep gray nuclei. Additionally, the proportional grid system could be used as a tool to study functional localization in different patients. Using the proportional grid system and direct and indirect localization principles, the patient's brain anatomy could be mapped and assessed with electroencephalography recordings to create presurgical hypotheses for placement of SEEG depth electrodes. Once electrodes were implanted according to the presurgical plan, spontaneous seizures and different brain structures could be recorded and analyzed. ${ }^{31,32)}$ Talairach and Bancaud's new technique offered the possibility of studying three-dimensional (3D) seizure patterns and their relationship with clinical features using longer and serial recordings. ${ }^{33)}$

In addition to Talairach's original stereotactic system, other neurosurgeons across the world were creating their own frames. Standard stereotactic technique previously used frames with simple orthogonal systems to direct electrodes perpendicularly into the skull and allow three degrees of freedom. Straight orthogonal approaches may be limiting for subcortical targets, so newer frames were created to include angular adjustment of electrodes. However, increased flexibility meant more complicated calculations were required to position electrodes correctly. Therefore, neurosurgeons like Lars Leksell created arc-quadrant systems using non-orthogonal paths. In this system, electrodes are directed along paths equal to the radius of a sphere where the tangent of an arc rotates around the vertical axis and the quadrant rotates around the horizontal axis. The Todd-Wells frame also uses an arc system but has the patient mobile within a fixed arc frame. ${ }^{34,35)}$

\section{Technical features}

SEEG is individualized for each patient using anatomo-electro-clinical (AEC) preimplantation hypotheses which help correlate seizures with possible lesion location. Because of this, the preimplantation hypotheses are vital for placement of electrodes and interpretation of recordings to identify the EZ. Depth electrodes should be placed in areas of suspected anatomic lesion, alternative but less likely hypothesized areas of EZ, areas of seizure onset, and the surrounding areas of spread that occur before and after seizure activity. Other diagnostic methods, such as MRI, PET, SPECT, may also be used to identify focal lesions relating to the EZ, areas of hypometabolism to verify EZ location, and regions of hyperperfusion during seizure activity, respectively. ${ }^{22)}$ A hypothesized 3D network for EZ localization is generated using a combination of non-invasive tests, clinical seizure manifestation, patient history, and risk factors. ${ }^{30)}$ Once planning is completed, depth electrodes are implanted using standard stereotactic technique or robotic devices.

In modern practice, a standard frame-based device, such as Todd Wells or Leksell frame, is typically used for implanting depth electrodes using three stages: stereotactic localization, digital angiography and MRI scan integration, and electrode implantation after adjusting the stereotactic frame. These steps can also be modified into a simpler procedure by implanting depth electrodes using 3D gadolinium-enhanced MRI scans with or without CT angiography. ${ }^{28)}$ This frame-based technique is advantageous, because it can provide electrode delivery to a specific target with $<3 \mathrm{~mm}$ accuracy. ${ }^{36)} \mathrm{A}$ frameless technique can also be used, which offers increased patient comfort, easier use, and more flexibility for preoperative planning while producing comparable results with standard frame-based techniques. ${ }^{37)}$ Recently, the use of robot assistance with both frame-based and frameless methods has been shown to increase accuracy even further while reducing 
time of implantation and the need for frame adjustments. ${ }^{32}$ Once the electrodes are implanted, electrocorticography samples are obtained from each electrode to ensure proper functioning, and electrode positions are confirmed with CT scan or intraoperative skull X-ray. Electrode stimulation is used to map brain functions and determine which electrodes correspond to seizure activity. This information is interpreted using the 3D recordings from depth electrodes and variable distances between electrodes to localize the EZ. ${ }^{38)}$

\section{Comparison with SDE}

In the United States, SDE has been the main choice for seizure mapping for decades. It involves performing a craniotomy or creating burr holes to create a subdural space to place electrode grids, which can record electrical activity and localize seizures on the cortical surface. SDE arrays can be customized with multiple rows and varying shapes and sizes for individual patients. Electrode arrays can vary in placement with 2-10 electrodes per row and 2-8 rows. They can be placed in many locations, such as the temporal, frontal, and interhemispheric regions. However, they are not ideal for recording activity in highly vascularized areas, such as the insula or Sylvian fissure. Many electrodes can be placed in the brain, but there may be a higher risk of infection due to the larger craniotomy and communication to the external space than in SEEG. Because the electrodes are fixed in one place, seizure activity could be missed if it occurs in the distance between electrodes. Therefore, a limitation of SDEs is that they can only record activity in an area restricted by craniotomy. ${ }^{39)}$ SDE is excellent at localizing seizures on the cortical surface but can only sample sulci and deeper brain structures with the use of depth electrodes. In comparison, SEEG provides sparse superficial coverage, but can localize seizures with improved targeting of deeper tissue and record nearly every cerebral structure..$^{10,38,40)}$

\section{Outcomes}

The incorporation of SEEG and SDE into clinical practice has brought functional, safety, and neurological outcomes that can be observed in the treatment of DRE patients. Large cohort and observational studies have been conducted comparing the two techniques, each suggesting certain implications that will allow for the comparison of SEEG and SDE as a treatment modality. Herein, SEEG and SDE will be compared based on morbidity, postoperative deficits, recovery time, improvement in seizure frequency, surgical blood loss, length of surgery, hospital length of stay, and patient psychological impact.

On the basis of morbidity, SEEG has been characterized to have lower morbidity rates than SDE usage. In a recent meta-analysis study, 1973 SEEG patients were compared to 2036 SDE patients with the results showing a $4.8 \%$ morbidity rate among SEEG use, compared to the $15.5 \%$ among the SDE patients. ${ }^{41)}$ Much of this disparity has been associated with the percutaneous insertion method tied with SEEG, compared to the more intolerable and further invasive method of a craniotomy that is required for SDE placement. With regard to recovery, SEEG patients tend to recover within 24-48 hours since unnecessary craniotomies are not performed. SDE procedures that require a craniotomy will typically endure a 4- to 8-week recovery time. Moreover, the procedure for an SEEG is estimated at 4 hours while the more invasive SDE method may require at least 3-5 hours of surgery. ${ }^{42,43)}$ Additionally, SEEG has minimal to no surgical blood loss, with the average estimated blood loss per SEEG implantation procedure is estimated at only $5 \mathrm{~cm}^{3}{ }^{44)}$ Conversely, the surgical blood loss in SDE procedures is dependent on the size and number of grids implanted. ${ }^{45}$ Should a craniotomy be performed, the average blood loss is estimated at 3.2 units. ${ }^{46)}$ In terms of hospital stay, SEEG and SDE are similarly hospitalized for 1-2 weeks' post-surgery for monitoring. ${ }^{42)}$ However, SDE patients may experience longer hospital stays should any operative complications manifest (Table 1).

While both procedures are relatively safe, postoperative deficits have been characterized from either method. The most commonly reported SEEG complications are superficial infection, electrode breakage, and the possibility of intracranial hemorrhage. Another meta-analysis study investigated the postoperative complications of SEEG treatment in a sample of 2624 patients, revealing an infection prevalence of $0.8 \%$, with cerebral abscess being the most common type of infection. ${ }^{10)}$ In addition, the same study revealed a $1.0 \%$ hemorrhage prevalence, with specifically intracranial hemorrhage being the most common type at $0.7 \%{ }^{10)}$ The risk of intracerebral hemorrhage is also higher in SEEG. ${ }^{2)}$ Transient neurological deficits were presented at a $0.6 \%$ prevalence among SEEG patients while permanent deficits were also at a $0.6 \%$ prevalence, yet their cause may not be directly correlated to the procedure itself. ${ }^{10)}$ Among pediatric patients, a further meta-analysis revealed that the most common neurological deficit from SEEG implantation was temporary hemiparesis. Other complications can include temporary aphasia, dysphagia, facial 
Table 1 Comparison of the postoperative complications following a meta-analysis of SEEG and SDE administration

\begin{tabular}{lcc}
\hline & SEEG & SDE \\
\hline Neurological infection prevalence & $0.8 \%$ & $2.3 \%$ \\
Intracranial hemorrhage prevalence & $0.7 \%$ & $4.1 \%$ \\
Neurological deficit prevalence (transient and permanent combined) & $1.2 \%$ & $4.6 \%$ \\
Number of deaths & $0.3 \%$ & $0.3 \%$ \\
\hline
\end{tabular}

Data provided by Katz and Abel (2019) ${ }^{10)}$ SDE: subdural electrode, SEEG: stereoelectroencephalography.

weakness, and homonymous hemianopsia. ${ }^{47)}$ Conversely, SDE has similar postoperative deficits but at higher prevalence. In all, 2542 SDE patients were analyzed, with the findings showing that SDE patients had a $2.3 \%$ prevalence for infection while intracranial hemorrhage had a $4.0 \%$ prevalence. ${ }^{10)}$ Furthermore, SDE patients revealed a $4.6 \%$ prevalence for neurological deficits, with very few being permanent. However, cerebrospinal fluid (CSF) leaks were one of the more adverse effects of this procedure with a rate of $12.1 \% .^{10)}$ This is explained due to the elevated intracranial pressure when SDE grids occupy space, or even due to complications that arise from the risk factors associated with craniotomy procedures. Nevertheless, both SEEG and SDE procedures reported five deaths in this study, with SDE reporting 10 hardware complications compared to the 11 from SEEG. ${ }^{10)}$

In terms of seizure improvement, both procedures show relative success. In a recent study observing the results of SEEG among viral encephalitis patients, 8/10 patients showed some level of seizure control. The results showed 3/10 patients becoming seizure free with a further five showing Engel outcomes of class II (rare disabling seizures) or III (worthwhile improvement). ${ }^{48)}$ In a further study, 106 out of 108 SEEG patients $(58 \%)$ underwent resection, with 26 out of the $81(32 \%)$ followed- up patients remaining seizure free. ${ }^{49)}$ With SDE studies, surgical resection was performed in 84 patients, with $70 \%$ experiencing Engel class I freedom from seizures. ${ }^{50)}$ In a direct meta-analysis study comparing the two methods, Engel class I or II were observed in 57 of 75 SEEG cases $(76.0 \%)$ and 59 of 108 SDE cases (54.6\%) among patients undergoing resection or ablation. ${ }^{40)}$ The analysis continued with only non-lesional cases, which showed positive seizure outcomes in $69.2 \%$ (27/39) of SEEG cases and $34.6 \%(9 / 26)$ of SDE cases. Moreover, when all patients were evaluated, and not just the ones undergoing definitive procedures, the positive seizure outcomes of both methods were similarly comparable at $47.1 \%$ and $42.4 \%$ for SEEG and SDE, respectively. ${ }^{40)}$
SEEG and SDE also reveal neuropsychiatric effects following their application. Although trends in psychosocial behavior vary regarding the subject, there are noticed psychological patterns in the treatment of epilepsy patients. In particular, SDE subjects have revealed rare instances of memory loss, inability to create new memories, auditory impairments, and mental fatigue. ${ }^{39)}$ However, the SDE procedure did not report significant changes to anxiety and depression levels following implantation. Moreover, preoperative fear and anxiety levels may be exhibited among SDE patients prior to a craniotomy, adding an element of psychological stress to the procedure. ${ }^{51)}$ On the other hand, psychological studies of patients' post-SEEG implantation is very limited. SEEG procedures may not present preoperative fear or anxiety, yet the prospect of being seizure free may not always lead to positive psychological benefits. While there are many patients that report improvement in their mental health following seizure freedom, there are other cases where the "burden of normality" can cause consequences that are as limiting as seizures themselves. ${ }^{52)}$ This complex pattern in epilepsy care should considered when treating patients with signs of mental health fragility.

\section{Discussion of Outcomes}

Both SEEG and SDE are feasible techniques in identifying seizure localization and investigating the pathology of epilepsy manifestations. However, modern studies reveal that SEEG is a more favorable technique due to its lower rates of infection, hemorrhage, and neurological deficits. SEEG reveals itself as a safer alternative to SDE, with SDE implantation containing the added risk of CSF leaks, higher morbid outcomes, and increased possibility of abnormal postoperative risks. Additionally, the fact that SEEG is minimally invasive allows for lower surgical blood loss, lower recovery times, and a lower probability of operative complications. Moreover, the simplicity of electrode removal within 
Table 2 A comparison of the clinical benefits and drawbacks between SEEG and SDE implantation

\begin{tabular}{|c|c|c|}
\hline & SEEG & SDE \\
\hline Safety & $\begin{array}{l}\text {-Lower rates of infection, hemorrhage, } \\
\text { neurological deficits, and morbidity }\end{array}$ & $\begin{array}{l}\text {-Increased rates of infection, hemorrhage, } \\
\text { neurological deficits, and morbidity }\end{array}$ \\
\hline Invasiveness & $\begin{array}{l}\text {-Faster procedure } \\
\text {-Lower hospital length of stay } \\
\text {-Simple electrode removal }\end{array}$ & -SDE commits patients to two craniotomies \\
\hline Versatility & $\begin{array}{l}\text {-Bilateral hemisphere investigations } \\
\text {-Analysis of deeper cerebral tissue } \\
\text {-Can record nearly every cerebral structure } \\
\text {-Useful for patients with temporal lobe epilepsy, } \\
\text { insular epilepsy, hypothalamic hamartoma }\end{array}$ & -Lacks the extensiveness and scope of SEEG \\
\hline Efficacy & -Higher rates of seizure control than SDE & -Lower rates of seizure control that SEEG \\
\hline
\end{tabular}

SEEG: stereoelectroencephalography, SDE: subdural electrode.

SEEG adds further benefit to its application. SDE procedures require patients to commit to two craniotomies, one for placement and one for extraction, while SEEG electrodes can be easily removed within 15 minutes without further hospital stay. The combination of these simplicity and safety benefits has led to SEEG's vast popularity for epilepsy treatment (Table 2).

SEEG also reveals better functional outcomes, with greater instances of seizure freedom and control compared to SDE studies. SEEG shows greater Engel class I and II rates among both general and nonlesional cases, highlighting its vast treatment potential. SEEG techniques can also allow for bilateral hemisphere monitoring at greater cerebral foci precision, whereas most SDE methods are limited to one hemisphere. This means SEEG can be further used to investigate TLE, insular epilepsy, and hypothalamic hamartoma while monitoring deeper tissue at higher anatomical accuracy. However, modified SDE methods can be used bilaterally. Moreover, SEEG is the sole method that allows for essential 3D information about the spatial and temporal organization of the ictal discharge. ${ }^{53}$ However, despite the advantages that SEEG has over SDE, SDE methods do have scenarios that make them preferable for cerebral monitoring. SDE provides higher density and more standardized two-dimensional (2D) maps of the outer cerebral surface, which make them more desirable when analyzing a unilateral superficial neocortical focus. ${ }^{38)}$ This can allow for more detailed extra-operative functional mapping, especially when seizure onset is near the eloquent cortex. In certain cases, both methods are incorporated, such as the case when SEEG has found cortical onset but the boundaries of the seizure onset zone and eloquent cortex remain unknown. ${ }^{38)}$ However, when evaluating DRE, SEEG should be used as the contributive tool for treatment for the majority of cases. Many SEEG studies have effectively located the EZ of patients suffering from pharmacoresistant epilepsy leading to positive outcomes in seizure recovery. A recent SEEG study located the EZ zone of $92 \%$ of the presented cases leading to seizure freedom in two-third of cases and seizure reduction in one-third of cases. ${ }^{38)}$ This indicates the efficacy of SEEG in treating DRE patients while also supporting its role in monitoring epilepsy manifestations.

\section{Conclusion}

After examining the outcomes of SEEG and SDE, it is evident that SEEG is the preferred method for localizing the EZ prior to epilepsy surgery. Observational data suggest that SEEG is safer than SDE, with lower rates of morbidity, infection, and other post-procedural complications. In addition, SEEG also provides 3D bihemispheric imaging and targets deeper cerebral tissue, making it a more informative tool than SDE. All of these benefits contribute to a more positive patient experience without the risk factors associated with SDE. However, in instances of locating seizures on the cortical surface, SDE may be the preferred method. Nevertheless, further studies are still needed to examine which technique is more advantageous in specific clinical cases. This will allow for a broadened understanding of the application of these techniques and guide neurosurgeons to effectively and accurately treat epilepsy patients. Moreover, further studies are also needed to assess the efficacy and pitfalls of robot-assisted SEEG, as this technique may be revolutionary to mainstream epilepsy care. Limitations such as the 
psychological impact of SEEG and SDE also need further evaluation since they could be significant factors during the presurgical assessment. Lastly, the establishment of a pre-determined algorithm for the evaluation of DRE patients must be determined to lower the lag-time between the diagnosis and surgical referral and ultimately provide better outcomes for DRE patients.

\section{Conflicts of Interest Disclosure}

The authors report no conflict of interest concerning the materials or methods used in this study or the findings specified in this paper.

\section{References}

1) Beghi E: The epidemiology of epilepsy. Neuroepidemiology 54: 185-191, 2020

2) Zack MM, Kobau R: National and state estimates of the numbers of adults and children with active epilepsy - United States. 2015. MMWR Morb Mortal Wkly Rep 66: 821-825, 2017

3) Brodie MJ, Shorvon SD, Canger R, et al.: Commission on European Affairs: appropriate standards of epilepsy care across Europe.ILEA. Epilepsia 38: 1245-1250, 1997

4) Kwan P, Brodie MJ: Early identification of refractory epilepsy. $N$ Engl J Med 342: 314-319, 2000

5) Anyanwu C, Motamedi GK: Diagnosis and surgical treatment of drug-resistant epilepsy. Brain Sci 8: 49, 2018

6) Wiebe S, Blume WT, Girvin JP, Eliasziw M: Effectiveness and Efficiency of Surgery for Temporal Lobe Epilepsy Study Group: A randomized, controlled trial of surgery for temporal-lobe epilepsy. $N$ Engl J Med 345: 311-318, 2001

7) Lowe AJ, David E, Kilpatrick CJ, et al.: Epilepsy surgery for pathologically proven hippocampal sclerosis provides long-term seizure control and improved quality of life. Epilepsia 45: 237-242, 2004

8) Elliott RE, Bollo RJ, Berliner JL, et al.: Anterior temporal lobectomy with amygdalohippocampectomy for mesial temporal sclerosis: predictors of long-term seizure control. J Neurosurg 119: 261-272, 2013

9) Tanriverdi T, Olivier A, Poulin N, Andermann F, Dubeau F: Long-term seizure outcome after mesial temporal lobe epilepsy surgery: corticalamygdalohippocampectomy versus selective amygdalohippocampectomy. J Neurosurg 108: 517-524, 2008

10) Katz JS, Abel TJ: Stereoelectroencephalography versus subdural electrodes for localization of the epileptogenic zone: what is the evidence? Neurotherapeutics 16: 59-66, 2019

11) Avanzini G, Tassi L: Epilepsy surgery in Italy. In: Luders HO, editor. Textbook of epilepsy surgery. London, CRC Press, 2008. p. 54-58
12) Dawda Y, Ezewuzie N: Epilepsy: clinical features and diagnosis. Clin Pharm 2: 86-88, 2010

13) Sander JW: The natural history of epilepsy in the era of new antiepileptic drugs and surgical treatment. Epilepsia 44 Suppl 1: 17-20, 2003

14) Beghi E, Giussani G, Sander JW: The natural history and prognosis of epilepsy. Epileptic Disord 17: 243-253, 2015

15) Kwan P, Sander JW: The natural history of epilepsy: an epidemiological view. J Neurol Neurosurg Psychiatry 75: 1376-1381, 2004

16) Kwan P, Arzimanoglou A, Berg AT, et al.: Definition of drug resistant epilepsy: consensus proposal by the ad hoc task force of the ILAE commission on therapeutic strategies. Epilepsia 51: 1069-1077, 2010

17) Sheng J, Liu S, Qin H, Li B, Zhang X: Drug-resistant epilepsy and surgery. Curr Neuropharmacol 16: $17-28,2018$

18) Laxer KD, Trinka E, Hirsch LJ, et al.: The consequences of refractory epilepsy and its treatment. Epilepsy Behav 37: 59-70, 2014

19) Jette N, Reid AY, Wiebe S: Surgical management of epilepsy. CMAJ 186: 997-1004, 2014

20) Engel J: Evolution of concepts in epilepsy surgery. Epileptic Disord 21: 391-409, 2019

21) Solli E, Colwell NA, Say I, et al.: Deciphering the surgical treatment gap for drug-resistant epilepsy (DRE): a literature review. Epilepsia 61: 1352-1364, 2020

22) Englot DJ: A modern epilepsy surgery treatment algorithm: Incorporating traditional and emerging technologies. Epilepsy Behav 80: 68-74, 2018

23) Kwan P, Arzimanoglou A, Berg AT, et al.: Definition of drug resistant epilepsy: consensus proposal by the ad hoc task force of the ILAE commission on therapeutic strategies. Epilepsia 51: 1069-1077, 2010

24) Choi H, Carlino R, Heiman G, Hauser WA, Gilliam FG: Evaluation of duration of epilepsy prior to temporal lobe epilepsy surgery during the past two decades. Epilepsy Res 86: 224-227, 2009

25) LoPinto-Khoury C, Sperling MR, Skidmore C, et al.: Surgical outcome in PET-positive, MRI-negative patients with temporal lobe epilepsy. Epilepsia 53: 342-348, 2012

26) Foiadelli T, Lagae L, Goffin K, et al.: Subtraction Ictal SPECT coregistered to MRI (SISCOM) as a guide in localizing childhood epilepsy. Epilepsia Open 5: 61-72, 2020

27) Oliveira Young C, Etchbehere ECSC, Souza EM, et al.: Clinical usefulness of SISCOM-SPM compared to visual analysis to locate the epileptogenic zone. Front Neurol 11: 467,2020

28) Iida K, Otsubo H: Stereoelectroencephalography: indication and efficacy. Neurol Med Chir (Tokyo) 57: 375-385, 2017

29) Bancaud J, Dell MB: Technics and method of stereotaxic functional exploration of the brain structures in man (cortex, subcortex, central gray nuclei). Rev Neurol (Paris) 101: 213-227, 1959

30) Gonzalez-Martinez JA: The stereo-electroencephalography: the epileptogenic zone. J Clin Neurophysiol 33: 522-529, 2016 
31) Harary M, Cosgrove GR: Jean Talairach: a cerebral cartographer. Neurosurg Focus 47: E12, 2019

32) González-Martínez J, Bulacio J, Thompson S, et al.: Technique, results, and complications related to robotassisted stereoelectroencephalography. Neurosurgery 78: 169-180, 2016

33) Reif PS, Strzelczyk A, Rosenow F: The history of invasive EEG evaluation in epilepsy patients. Seizure 41: 191-195, 2016

34) Zhang G, Chen G, Meng D, et al.: Stereoelectroencephalography based on the Leksell stereotactic frame and neurotech operation planning software. Medicine 96: e7106, 2017

35) Maciunas RJ, Galloway RL, Latimer JW: The application accuracy of stereotactic frames. Neurosurgery 35: 682-694; discussion 694-695, 1994

36) Cardinale F, Cossu M, Castana L, et al.: Stereoelectroencephalography: surgical methodology, safety, and stereotactic application accuracy in 500 procedures. Neurosurgery 72: 353-366; discussion 366, 2013

37) Verburg N, Baayen JC, Idema S, et al.: In vivo accuracy of a frameless stereotactic drilling technique for diagnostic biopsies and stereoelectroencephalography depth electrodes. World Neurosurg 87: 392-398, 2016

38) Youngerman BE, Khan FA, McKhann GM: Stereoelectroencephalography in epilepsy, cognitive neurophysiology, and psychiatric disease: safety, efficacy, and place in therapy. Neuropsychiatr Dis Treat 15: 1701-1716, 2019

39) Lesser RP, Crone NE, Webber WRS: Subdural electrodes. Clin Neurophysiol 121: 1376-1392, 2010

40) Tandon N, Tong BA, Friedman ER, et al.: Analysis of morbidity and outcomes associated with use of subdural grids vs stereoelectroencephalography in patients with intractable epilepsy. JAMA Neurol 76: 672-681, 2019

41) Yan H, Katz JS, Anderson M, et al.: Method of invasive monitoring in epilepsy surgery and seizure freedom and morbidity: a systematic review. Epilepsia 60: 1960-1972, 2019

42) Epilepsy Surgery, University of Michigan Neurosciences; 2020; https://www.uofmhealth.org/conditionstreatments/brain-neurological-conditions/epilepsysurgery. (Accessed on 2020 September 22)
43) Craniotomy. Mayfield brain \& spine. https://mayfieldclinic.com/pe-craniotomy.htm. (Accessed on 2020 September 22)

44) Harrison P: SEEG an option for difficult-to-localize epilepsy in kids. Medscape, 2014; https://www. medscape.com/viewarticle/832061 (Accessed on 2020 September 22)

45) Onal C, Otsubo H, Araki T, et al.: Complications of invasive subdural grid monitoring in children with epilepsy. J Neurosurg 98: 1017-1026, 2003

46) Sitohang D, R. AM, Arif M: Estimated blood loss in craniotomy. Nusantara Med Sci J 1: 143-145, 2016

47) Sacino MF, Huang SS, Schreiber J, Gaillard WD, Oluigbo CO: Is the use of stereotactic electroencephalography safe and effective in children? A meta-analysis of the use of stereotactic electroencephalography in comparison to subdural grids for invasive epilepsy monitoring in pediatric subjects. Neurosurgery 84: 1190-1200, 2019

48) Liu YO, Zhou WJ, Hong B, Zhao T, Wang YF: Surgical outcomes in patients with epilepsy after viral encephalitis: contribution of SEEG study. BMC Neurol 19: 165, 2019

49) Steriade C, Martins W, Bulacio J, et al.: Localization yield and seizure outcome in patients undergoing bilateral SEEG exploration. Epilepsia 60: 107-120, 2019

50) Mullin JP, Sexton D, Al-Omar S, Bingaman W, Gonzalez-Martinez J: Outcomes of subdural grid electrode monitoring in the stereoelectroencephalography era. World Neurosurg 89: 255-258, 2016

51) Hejrati N, Spieler D, Samuel R, Regli L, Weyerbrock A, Surbeck W: Conscious experience and psychological consequences of awake craniotomy. World Neurosurg 129: e381-e386, 2019

52) Wilson S, Bladin P, Saling M: The "burden of normality": concepts of adjustment after surgery for seizures. J Neurol Neurosurg Psychiatry 70: 649-656, 2001

53) Cardinale F, Cossu M: SEEG has the lowest rate of complications. J Neurosurg 122: 475-477, 2015

Corresponding author: Brian Fiani, DO

Desert Regional Medical Center, 1150 N. Indian Canyon Dr., Palm Springs, CA 92262, USA. e-mail: bfiani@outlook.com 